\title{
Cryo-Transmission Electron Microscopy in Korean Society of Microscopy
}

\author{
Sung Sik Han* \\ School of Life Sciences and Biotechnology, Korea University, Seoul 02841, Korea
}

*Correspondence to:

Han SS,

Tel: +82-2-3290-3924

Fax: +82-2-3290-3424

E-mail: sshan@korea.ac.kr

Received November 29, 2017

Revised December 4, 2017

Accepted December 5, 2017
Dr. Jacques Dubochet, Dr. Joachim Frank, and Dr. Richard Henderson received the 2017 Nobel Prize for Chemistry for their efforts to develop effective ways to obtain highresolution three-dimensional images of biomolecules using cryo-electron microscopy. Congratulations to the Nobel Prize in the field of electron microscopy, I will explain the scientific contributions of the three winners and introduce the role of cryo-electron microscopy (including cryo technology) in biology.

Key Words: Cryo-transmission electron microscopy, Nobel Prize, Cryo-fixation, High resolution, Molecular structure
In the field of electron microscopy (EM), the Nobel Prize in physics was awarded in 1986 to Dr. Ernst Ruska of Germany for his fundamental work in electron optics and for designing the first electron microscope (and to Dr. Gerd Binnig and Dr. Heinrich Rohrer for their contributions to the design of the scanning tunneling microscope). Furthermore, in 2017, the Nobel Prize in chemistry was awarded in the same field. The chemistry prize winners were Dr. Jacques Dubochet (University of Lausanne, Switzerland), Dr. Joachim Frank (Columbia University, New York, NY, USA), and Dr. Richard Henderson (MRC Laboratory of Molecular Biology, Cambridge, United Kingdom). All three focused on "developing cryo-electron microscopy for the high-resolution structure determination of biomolecules in solution." Therefore, first, I completely welcome to their winnings, as I had a lot of good achievements using cryo-transmission electron microscopy (TEM).

A brief analysis of the accomplishments mentioned above indicates that the study of the development of the cryoEM field that verifies the high-resolution crystal structure of biomolecules can be divided into three parts: cryo-EM, biomolecule, and high resolution.

Dr. J. Dubochet used quick-freezing methods in the early 1980s to solve the problem of drying samples (Dubochet \& McDowall, 1981; Dubochet et al., 1982, 1988). The development of this test method allowed the molecular state, even in its natural state, to be preserved in a liquid state. Dr. R. Henderson extended cryo-TEM to biomolecule observations; he developed a cryo-EM study to observe biomolecules by presenting bacterial molecules at atomic level resolution (Henderson \& Unwin, 1975). J. Frank of Germany developed an image signal processing technology that allows only the shape of particles to be arranged in the process of three-dimensional (3D) realization of the cryo-TEM images obtained by the two teams (Frank, 1975; Frank et al., 1991). Today, it is common to realize $3 \mathrm{D}$ images of viruses or protein parts, which I think is due to the source technology that was created by the collaboration of these three people.

Thanks to their efforts, the field of cryo-EM or cryo-high voltage electron microscopy (HVEM) is emerging not only in biology but also in various study fields such as engineering and agriculture.

Nonetheless, I will discuss from a biology standpoint. Firstly, I am familiar with Dr. J. Dubochet as I have seen him three or four times at meetings or conferences in Europe, and personally, I still vividly remember the passionate lecture he had given at the IMC 16 in Sapporo, Japan. As for Dr. J. Frank, readers had been deeply impressed by the guide he provided in his famous books, including "Three-Dimensional Electron Microscopy of Macromolecular Assemblies”

(a) This is an open-access article distributed under the terms of the Creative Commons Attribution Non-Commercial License (http://creativecommons.org/licenses/by-nc/4.0) which permits unrestricted noncommercial use, distribution, and reproduction in any medium, provided the original work is properly cited.

Copyrights @ 2017 by Korean Society of Microscopy 
(Frank, 1996), another book with the same title, "ThreeDimensional Electron Microscopy of Macromolecular Assemblies: Visualization of Biological Molecules in Their Native State" (Frank, 2006), and the most decisive one titled, "Electron Tomography, Methods for Three-Dimensional Visualization of Structure in the Cell" (Frank, 2005). From which, I have studied the following chapters in detail: "Introduction: Principles of Electron Tomography (Joachim Frank)," "Chapter 2: Electron Tomography of Frozenhydrated Sections of Cells and Tissues (Michael Marko, Chyong-Ere Hsieh and Carmen A. Mannella)," and "Chapter 4: Cryotomograghy: Low-Dose Automated Tomography of Frozen-Hydrated Specimens (Abraham J. Koster and Montserrat Barcena)." I also have memories of attempting to understand all the books, including the image processing parts, with the help Professor Ye, Jong Chul's team of KAIST (Korea Advanced Institute of Science and Technology) and Professor Kim, Dong Sik's of the Hankuk University of Foreign Studies in 2006. It was very difficult for me, as a biologist, to understand computer engineering or other fields of engineering.

From the perspective of a biologist, I find that the current cryo-EMs have made it all possible (Fig. 1): whether it pertains to direct observations with a cryo-EM after processing by plunge-freezing in a living state (Vitrobot can be purchased or be fabricated), slam-freezing (without chilling, totally different from plunge-freeing), or high-pressure freezing
(HPF); whether it is observation with a cryo-EM after cryosection or cryo-electron tomography (ET) (the frozen sample are imaged as they are tilted different angles by $1^{\circ}$ or $2^{\circ}$ ); or whether it is $3 \mathrm{D}$ observations (representative examples are viruses and single particle analysis).

This can be explained in other words. (1) For a cryo-electron microscopy, taking my study as an example, we did structural analysis by cryo-TEM after elution of each spot, which included separation of anti-microbial proteins that were generated during the Bombyx mori immune reaction using one-dimensional (1D) electrophoresis and two-dimensional (2D) electrophoresis. Moreover, (2) Cell-membrane structures revealed and by cryo-TEM were mitochondrial proteins, Golgi apparatus associated proteins, nuclear-pore structures, and cell membrane-associated proteins. Furthermore, (3) Continuous repetitive structures, such as the formation of microtubules and neurotubules, flagella, and axoneme were analyzed to study brain function and structures. (4) Icosahedral structures of various viruses. (5) Special structures of bacteriophage. Acquisition software for these applications is well known, including Xplore 3D, TOM toolbox, TEMograpy, and SerialEM. More detailed information should be examined by individual researchers.

It can be inferred that there are two steps, i.e., the most basic cryo-fixation such as plunge-freezing and the subsequent computer work comprising particle picking, image enhancement, and projecting the image.

\section{Cryo-electron microscope workflow}

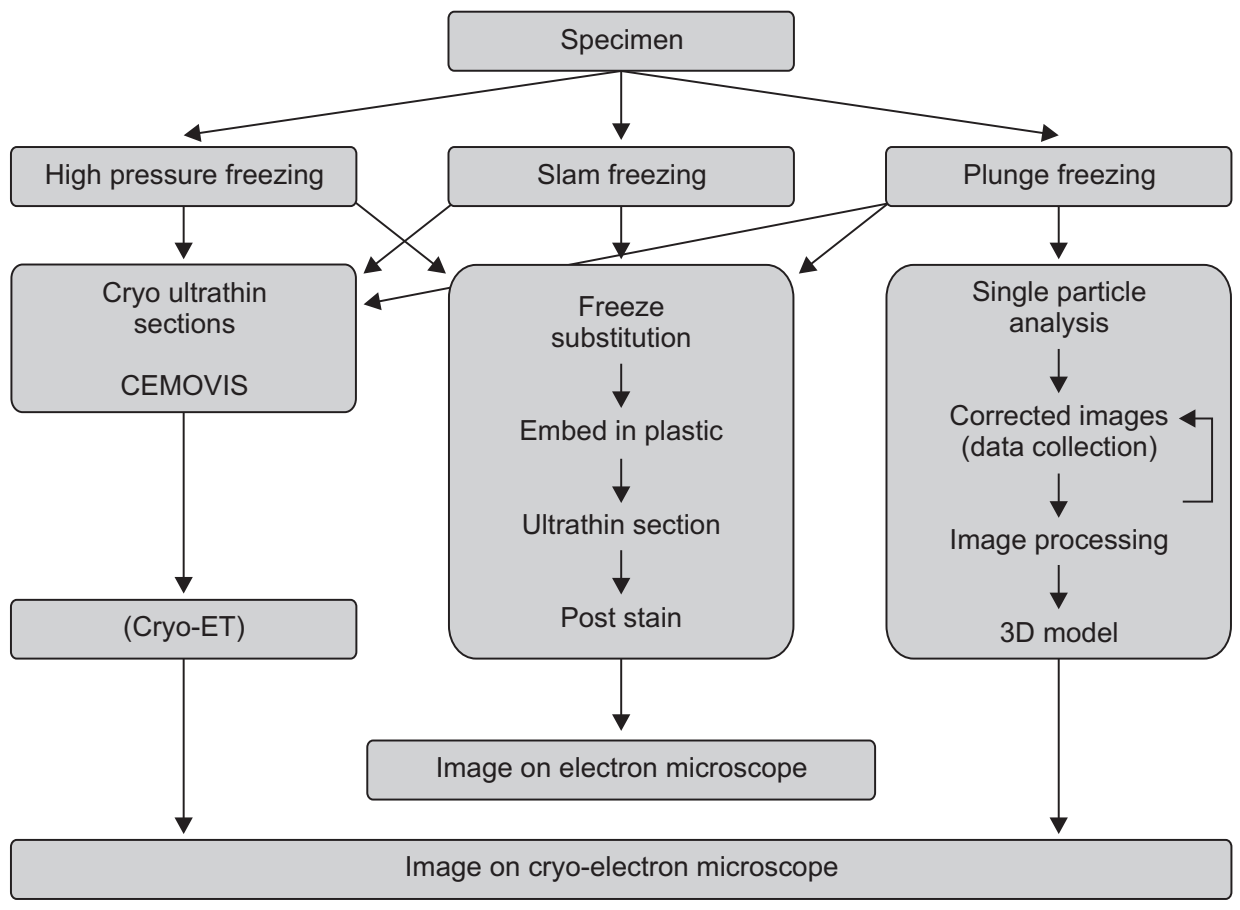

Fig. 1. Cryo-elecron microscope workflow. CEMOVIS, cryo-electron microscopy of vitreous sections; ET, electron tomography. 
In addition, J. Dubochet's cryo-electron microscopy of vitreous sections (CEMOVIS) can be used to perform cryofixation by slam-freezing or HPF. CEMOVIS is widely used, particularly at high resolutions.

In the biological field, what cannot be excluded from the most important micro-techniques in addition to cryo-fixation is the so-called Tokuyasu Cryo-Section and Immunolabeling, SDSdigested Freeze-Fracture Replica Labeling (SDS-FRL), and Correlative Light and Electron Microscopy (CLEM), which are well documented in the "Handbook of Cryo-Preparation Methods for Electron Microscopy” (Annie Cavalier, 2008).
And enzymatic activities such as "Demonstration of Alkaline Phosphatase and PAS", which belong to the cytochemistry, has been revealed one after another, but why can we not join in order to get better results? And if you are a biologist and have knowledge of electrical engineering or electronics, how about approaching CLEM with a different idea?

\section{CONFLICT OF INTEREST}

No potential conflict of interest relevant to this article was reported.

\section{REFERENCES}

Cavalier A, Spehner D, and Humbel B M (2008) Handbook of CryoPreparation Methods for Electron Microscopy (CRC Press, Boca Raton).

Dubochet J, Adrian M, Chang J J, Homo J C, Lepault J, McDowall A W, and Schultz P (1988) Cryo-electron microscopy of vitrified specimens. Quart. Rev. Biophys. 21, 129-228.

Dubochet J, Lepault J, Freeman R, Berriman J A, and Homo J C (1982) Electron microscopy of frozen water and aqueous solutions. J. Microsc. 128, 219-237.

Dubochet J and McDowall A W (1981) Vitrification of pure water for electron microscopy. J. Microsc. 124, 3-4.

Frank J (1975) Averaging of low exposure electron micrographs of nonperiodic objects. Ultramicroscopy 1, 159-162.
Frank J (1996) In Three-Dimensional Electron Microscopy of Macromolecular Assemblies, pp. xv-xvii (Academic Press, Burlington).

Frank J (2005) Electron Tomography, Methods for Three-Dimensional Visualization of Structure in the Cell (Springer, New York).

Frank J (2006) Three-Dimensional Electron Microscopy of Macromolecular Assemblies: Visualization of Biological Molecules in Their Native State (Oxford University Press, Oxford).

Frank J, Penczek P, Grassucci R, and Srivastava S (1991) Three-dimensional reconstruction of the $70 S$ Escherichia coli ribosome in ice: the distribution of ribosomal RNA. J. Cell Biol. 115, 597-605.

Henderson R and Unwin P N T (1975) Three-dimensional model of purple membrane obtained by electron microscopy. Nature 257, 28. 